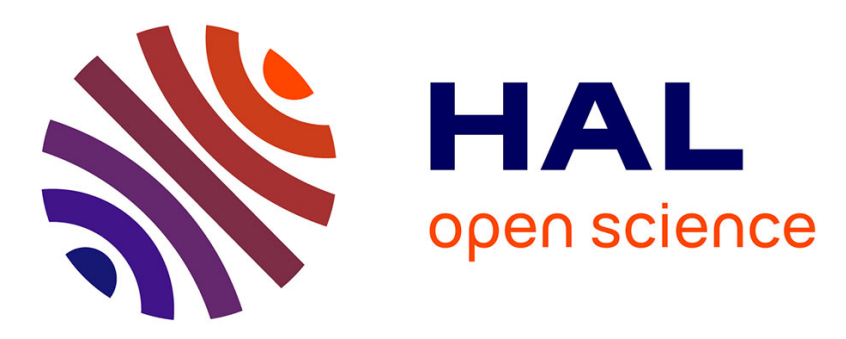

\title{
Femtosecond Broadband Frequency Switch of Terahertz Three-Dimensional Meta-Atoms
}

\author{
Paul Goulain, Anastasios Koulouklidis, Jean-Michel Manceau, Christina \\ Daskalaki, Bruno Paulillo, Kenneth Maussang, Sukhdeep Dhillon, Joshua \\ Freeman, Lianhe Li, Edmund Linfield, et al.
}

\section{To cite this version:}

Paul Goulain, Anastasios Koulouklidis, Jean-Michel Manceau, Christina Daskalaki, Bruno Paulillo, et al.. Femtosecond Broadband Frequency Switch of Terahertz Three-Dimensional Meta-Atoms. ACS photonics, 2021, 8 (4), pp.1097-1102. 10.1021/acsphotonics.0c01802 . hal-03244480

\section{HAL Id: hal-03244480 \\ https: / hal-univ-paris.archives-ouvertes.fr/hal-03244480}

Submitted on 12 Nov 2021

HAL is a multi-disciplinary open access archive for the deposit and dissemination of scientific research documents, whether they are published or not. The documents may come from teaching and research institutions in France or abroad, or from public or private research centers.
L'archive ouverte pluridisciplinaire HAL, est destinée au dépôt et à la diffusion de documents scientifiques de niveau recherche, publiés ou non, émanant des établissements d'enseignement et de recherche français ou étrangers, des laboratoires publics ou privés. 


\title{
Femtosecond broadband frequency switch of terahertz three-dimensional meta-atoms
}

\author{
Paul Goulain ${ }^{1}$, Anastasios D. Koulouklidis ${ }^{2}$, Jean-Michel Manceau ${ }^{1,}{ }^{*}$, Christina Daskalaki ${ }^{2}$, \\ Bruno Paulillo ${ }^{1 \#}$, Kenneth Maussang ${ }^{3+}$, Sukhdeep Dhillon ${ }^{3}$, Joshua Freeman ${ }^{4}$, Lianhe $\mathrm{Li}^{4}$, \\ Edmund H. Linfield ${ }^{4}$, Stelios Tzortzakis ${ }^{2,5,6}$, Raffaele Colombelli ${ }^{1}$ \\ ${ }^{1}$ Centre de Nanosciences et de Nanotechnologies (C2N), CNRS UMR 9001, Université Paris-Saclay, 91120 \\ Palaiseau, France \\ ${ }^{2}$ Institute of Electronic Structure and Laser (IESL), Foundation for Research and Technology - Hellas (FORTH), \\ P.O. Box 1527, GR-71110, Heraklion, Greece \\ ${ }^{3}$ Laboratoire de Physique de l'Ecole normales supérieure (ENS), Université PSL, CNRS, Sorbonne Universitë', \\ Université de Paris, F-75005 Paris, France \\ ${ }^{4}$ School of Electronic and Electrical Engineering, University of Leeds, Woodhouse Lane, Leeds LS9 2JT, United \\ Kingdom \\ ${ }^{5}$ Department of Materials Science and Technology, University of Crete, GR-71003, Heraklion, Greece \\ ${ }^{6}$ Science Program, Texas A\&M University at Qatar, P.O. Box 23874 Doha, Qatar
}

\begin{abstract}
:
We present the experimental demonstration of a sub-picosecond all-optical THz switch based on three-dimensional (3D) terahertz meta-atoms. Combining a special design of 3D meta-devices and the ultrafast dynamics of low temperature grown Gallium Arsenide, we can modulate the reflectance of the $\mathrm{THz}$ micro-cavities within 2.2 picoseconds. The device enables a $280 \mathrm{GHz}$ switch in resonance frequency within less than $200 \mathrm{fs}$. The switch back to the original resonance takes $800 \mathrm{fs}$. Experimental results show that the speed values are strongly convoluted by the $\mathrm{THz}$ probing field and thus the real switching times are even shorter, in the few $100 \mathrm{fs}$ range.
\end{abstract}

Keywords: metasurfaces, photoswitching, ultrafast modulation, carrier dynamics, 
From the early days when metamaterials were used for textbook demonstrations of novel electromagnetic (EM) response ${ }^{1-3}$, a natural course of action has been their hybridization with optoelectronic devices in view of implementing novel functionalities and improved performances. Meta-devices have been demonstrated in a vast number of research fields and over several spectral ranges of the EM spectrum ${ }^{4}$. In the Terahertz $(\mathrm{THz})$ range, there have been numerous works developing components that can for instance modulate amplitude and phase ${ }^{5-9}$ of an incoming laser beam. It is also of interest to combine the sub-wavelength resonators that compose the meta-materials themselves with emitters or detectors to benefit from their circuital properties and extreme confinement. In this respect, the combination of metasurfaces with quantum cascade structures in the mid-infrared (MIR) and $\mathrm{THz}$ range has been an active field of research ${ }^{10-14}$. In particular, it was recently shown to be a relevant approach to increase the operating temperature of quantum well infrared photodetector by a significant reduction of their active surface (and in turn dark current) while maintaining their photon capture efficiency ${ }^{15}$. Furthermore, in $\mathrm{THz}$ quantum electrodynamics, LC resonators have been proposed to test the bosonization limit with few electrons in strong coupling ${ }^{16}$. The strong coupling regime between light and matter is also interesting as it opens up several possibilities for the development of non-classical light emitters ${ }^{17-19}$, that could be envisioned even up to room temperature at $\mathrm{THz}$ frequencies with the use of graded alloy parabolic quantum well $(\mathrm{QW})^{20}$. In such regime, the coupling strength between light and matter is so strong that new quasi-particles named polaritons arise. An important figure of merit to enter in this regime of interaction is the overlap between the device active region and the EM field. As the latter one is extremely sub-wavelength sized for metamaterials, this is quite challenging. Furthermore, standard planar metamaterial configurations cannot be used as intersubband (ISB) transitions interact only with transverse magnetic (TM) polarized light. An alternative exists at GHz frequency with the use of transverse electric (TE) polarized Landau levels coupled with planar metastructures ${ }^{21}$. In fact, it has been successfully used to study sub-cycle non-adiabatic physics in a very recent publication ${ }^{22}$. Nevertheless, it remains incompatible with a room temperature outlook strategy, since Landau polaritons require cryogenic temperature (10K or less) and the use of an external magnetic field. Several three-dimensional (3D) architectures have been proposed in recent years ${ }^{23-26}$, that have led to the demonstration of the strong coupling regime with ISB transitions ${ }^{27-29}$. These 3D architectures are interesting as they offer relatively high overlap factor with a field polarization compatible with ISB transitions. Nevertheless, the integration of ultrafast switching functionality to these architectures remains a challenge.

Meta-structures are ideal tools to enable optoelectronic devices with fast switching functionalities. Usually, the switching process relies on the conductivity change of a photosensitive material placed at the vicinity of a capacitance within the LC circuit. Under illumination with a near infrared (NIR) light 
pulse, electron-hole pairs are created. This modifies the complex refractive index of the medium ideally leading to a short-circuit of the capacitive element. In turn, the overall capacitance of the circuit is modified along with the resonant frequency of the system. To this end, several photosensitive materials, which can operate at room temperature, have been explored at $\mathrm{THz}$ frequencies, with the most common ones being semiconductors, $2 \mathrm{D}$ and phase transition materials ${ }^{9}$. The faster modulation speeds of the overall switching cycle (ON / OFF) reported so far are in the range of tens of picoseconds 30-33.

In this letter, we report on the demonstration of a femtosecond switchable device based on threedimensional LC micro-resonators with an overall modulation speed of $2 \mathrm{ps}$. This is actually an upper limit, as the recorded response is convoluted with the probe pulse. We use the circuital properties of the system to implement a secondary in-series capacitance and change its conductivity under NIR ultrafast laser pulse illumination. We choose low-temperature grown (LT)-GaAs as the photoswitching material to benefit from its intrinsic ultrafast carriers excitation and recombination rate ${ }^{34-}$ 37. Using a state-of-the-art THz time domain spectrometer, we observe that the LC circuit can operate on two states whose resonance frequencies are separated by $280 \mathrm{GHz}$, and it is possible to switch it from one to the other with an optical control beam. A switching ON time of $200 \mathrm{fs}$ has been measured along with a recombination time (switch OFF) lasting $800 \mathrm{fs}$, both recorded values being limited by the probing system time resolution. The overall modulation cycle last for 2.2 picoseconds as the system remains in the main switched mode for 1.2 ps. These results constitute record timing values for a $\mathrm{THz}$ signal amplitude modulation.

A schematic of the experimental approach is presented in Figure 1a, along with an equivalent circuit representation of the device in Figure $1 \mathrm{~b}$. An array of LC cavities is fabricated on top of a host substrate (GaAs) on which a 2- $\mu$ m-thick LT-GaAs layer was grown. We have not performed a post-growth annealing step, to preserve a high density of trapping states and its ultra-fast (femtosecond) recombination times ${ }^{35}$. To optically access the ultra-fast switching layer, a ring opening (width of 1.4 $\mu \mathrm{m})$ is formed into the ground plane of each unit cell, implementing a photo-switchable capacitance within the circuit. The unit cells are repeated on a square $2 \mathrm{D}$ array of period $\Lambda=22 \mu \mathrm{m}$. The main capacitive section ( $\left.\mathrm{C}_{\text {cavity }}\right)$ of the circuit is made of a silicon dioxide $\left(\mathrm{SiO}_{2}\right)$ patch that is fabricated via plasma enhanced chemical vapour deposition (PECVD) followed by reactive ion etching (RIE). The radius of the patch cavity is $2.4 \mu \mathrm{m}$ with a height of $370 \mathrm{~nm}$, resulting in an extremely small effective confinement volume of $\mathrm{V}_{\text {eff }}=3 \cdot 10^{-6} \lambda_{0}^{3}$. Finally, the inductive section of the circuit is obtained using an original micro-fabrication technique that allows to form an air-bridge metallic antenna ${ }^{24}$. The confined electric field of the resonant mode along two different planes is depicted in Figure 1c,d. Most of the field is confined within the cavity section, with the electric field $\left(E_{z}\right)$ orthogonal to the plane. This 
confinement permits the interaction with ISB transitions in semiconductor quantum wells (QW), that are the dipoles commonly employed for achieving strong light-matter coupling in this frequency range 27.

The resonance frequency of the system is given by $1 / \sqrt{L C}$, where $C$ stands as the equivalent capacitance of the circuit, equal to $1 / C_{\text {eq. }}=1 / C_{\text {ring }}+1 / C_{\text {cavity. }}$ In turn, the expected photo-switched frequency will depend on the relative value of each capacitance and their respective balance. We calculated the value of each of them as a function of increasing patch radius while keeping the ringopening dimension constant. This leads to a crossing point in between these two values that marks the relative frequency shift of the switch (Figure 1e). The device can be engineered to exhibit either a red or a blue frequency shift of the photo-activated resonance. The value of the frequency shift is reported in the upper panel of Figure 1e. This level of flexibility is of interest as it permits to optimize the device design in accordance with the constraints coming from either the active material to be used and/or the available bandwidth of the measurement system. Note: an iso-frequency behaviour can be obtained by changing accordingly the value of the inductance, as thoroughly discussed in Ref. ${ }^{24}$. Figure If shows a Scanning Electron Microscope (SEM) image of a typical fabricated device. We have also fabricated complementary devices with no ring opening in the ground plane. Those devices, named LC resonators, allow us to measure the resonant frequency of the circuit with a single capacitance (see the SEM picture on the lower panel of Figure 1f). This represents the ideal configuration of a fully switched device, i.e. a device where the NIR pulse illumination has brought the permittivity of the LTGaAs close to the one of a metallic layer.

We have probed both devices using a $\mathrm{THz}$ time-domain spectrometer based on the two-colour filamentation generation scheme ${ }^{38}$, which provides broadband $\mathrm{THz}$ pulses with a spectral bandwidth that exceeds $20 \mathrm{THz}$. The THz electric-field transient is probed using an Air-Biased-Coherent-Detection ( $A B C D$ ) sampling technique ${ }^{38,39}$. ABCD offers a larger bandwidth detection (up to $20 \mathrm{THz}$ ) and can resolve faster transients than the classic electro-optic technique with non-linear crystals, although with less sensitivity. The probed LC micro-resonators are made of spatially separated lumped elements, and they present specific light coupling rules as discussed in Ref. ${ }^{24}$. The optimal configuration allows exciting both electric and magnetic dipoles of the device. Figure 2a shows the reflectance of both the $\mathrm{LC}$ resonator and the $\mathrm{LC}$ switch devices at an incidence angle of 45 degrees and TM light polarization. We have obtained it by applying a Fast Fourier Transform to the recorded THz electric field transients. The native resonance of the LC switch (green curve; no illumination) indicates a frequency of $2.36 \mathrm{THz}$. In black, we measured the resonance of the LC resonator control device with a fully metallic ground plane at $2.1 \mathrm{THz}$. It mimics the ideal case, where the ring-opening material reaches metallic 
conductivity values upon optical excitation with the fs laser pulse. This configuration would offer a 260 $\mathrm{GHz}$ frequency shift between the two eigen modes of the device.

The switching functionality along with its time dynamics have been assessed using a synchronized collinear NIR pump pulse ( $\lambda \sim 800 \mathrm{~nm}, 35 \mathrm{fs}$ duration). The diameter of the NIR beam was larger than the $\mathrm{THz}$ probe beam, ensuring uniform excitation over on the sample where we probed an estimated number of 31776 resonators. The collinearity prevents any further time delay that could be added by a geometrical mismatch of the two beams' wavefronts. Under illumination (blue line in Figure 2a), the circuit oscillates on a second resonance at a red-shifted frequency of $2.08 \mathrm{THz}$. This corresponds to a $280 \mathrm{GHz}$ frequency shift, slightly higher than the expected value from the control LC resonator sample (black line) but less pronounced. The difference in the observed photoinduced frequency shift compared to the net LC circuit is linked to the achievable level of photoinduced conductivity change of the LT-GaAs.

To confirm this, we performed experiments demonstrating that the maximum achievable conductivity under IR laser excitation reaches values that are lower than the metallic ones. Figure $2 b$ shows the recorded relative change of reflectivity $(\Delta R / R)$ on a bare $L T-G a A s$ wafer at different levels of fluence. For each fluence the THz reflectance spectrum is recorded and compared to the dark case without NIR illumination $\left(\Delta R / R=\left(R_{\text {illu. }}-R_{\text {dark }}\right) / R_{\text {dark }}\right)$. Data are shown in the spectral region of interest, here at $2.1 \mathrm{THz}$. One can see that above a fluence of $2 \mathrm{~mJ} / \mathrm{cm}^{2}$, the relative reflectivity is saturating, and no further change can be obtained with increasing fluence. The saturated reflectivity of the LT-GaAs surface is approximately half that of a gold surface.

Considering this fact, we performed a series of simulations by changing the conductivity of the ring capacitance within the LC switch device. Figure 2c shows the LC switch resonance for three different values of conductivity within the LT-GaAs layer. The conductivity values are chosen to fit the experimental data and are expressed in siemens per meter $(\mathrm{S} / \mathrm{m})$. The simulations confirms that our experimental observation corresponds to an intermediate case (blue curve) where the switch capacitance has not reached the permittivity of a metal yet (black curve). In turn, this leads to a damped response of the system and a lower contrast of the switched mode.

Let us now focus on the time dynamics of the device. Figure 3 shows a 2-dimensional map of the frequency switch as a function of the delay between the NIR pulse and the THz probe. One can observe that the main frequency is red shifted by $280 \mathrm{GHz}$. The overall process takes about 2.2 picoseconds to transition from the switched mode back to the initial state. Thanks to the high temporal resolution of the $A B C D$ technique, we can observe fine details within the overall cycle. For instance, one can observe a broadening of the initial mode prior to the switching process. The rise of the switched mode (ON 
process) occurs on an extremely short time of $200 \mathrm{fs}$. The switched mode lasts for about 1.2 ps prior to recombination of the excited carriers while the OFF modulation takes $800 \mathrm{fs}$ before reaching back to the initial frequency of the device at $2.4 \mathrm{THz}$.

Such details in the dynamics have been revealed thanks to the high-resolution $A B C D$ detection scheme. Indeed, if the $A B C D$ is replaced with electro-optic detection using a ZnTe crystal much longer time dynamics are observed due to the significantly smaller detection bandwidth. In figure $3 \mathrm{~b}$, we provide the bare time traces of the $\mathrm{THz}$ pulse recorded with both techniques. The $\mathrm{THz}$ pulse envelope when recorded with $A B C D(\sim 190 f s$ FWHM) is twice shorter than the one recorded with the ZnTe crystal ( 400fs FWHM). We have then probed the change of reflectivity as a function of the time delay with the NIR pump, on the bare LT-GaAs layer (right panel of Figure 3b). This change in reflectivity is recorded at the maximum of the $\mathrm{THz}$ pulse electric field and depicts the dynamic of excited carriers within the LT-GaAs layer. As expected from previous literature results, for both cases one can observe a sharp rise after the excitation of carriers followed by a slower exponential decay. In the case of $A B C D$ technique, both the rise ( $200 \mathrm{fs})$ and the decay times ( $\sim 00 \mathrm{fs}$ ) are twice faster than in the case of ZnTe crystal which illustrate that the recorded dynamic is clearly convoluted by the probe $\mathrm{THz}$ pulse duration. Thus, one can expect even shorter time features to emerge with this device. We further observe that the real electrical current of the LC circuit oscillates $1.2 \mathrm{ps}$ on the switched mode before decaying back to the natural frequency of the circuit. This waiting time is not present in the timeresolved reflectivity measurements on bare LT-GaAs (Fig. 3b, right panel), suggesting that this feature is peculiar to the LC circuit. We do not have a final explanation for this observation, which is beyond the scope of this paper, and that we will try to elucidate in a future work. However, the switching values reported here are compatible with the non-adiabatic timing needed for the observation of the dynamical Casimir effect, while the overall THz signal modulation speed (ON/OFF) compete with the ones from the literature.

In conclusion, we have demonstrated a meta-surface, made of an array of 3D THz meta-atoms that permits to switch between two resonant states on femtosecond timescales. The resonant frequencies of the two states are separated by $280 \mathrm{GHz}$. Our next step is to combine this ultrafast frequency switch with the ISB transition dipoles of parabolic quantum wells to operate in the strong coupling regime at room temperature ${ }^{20}$. We will avoid perturbing the active semiconductor cavity with the NIR pump pulse by illuminating the Lt-GaAs layer from the back, using a transparent substrate as recently demonstrated ${ }^{40}$. This system would constitute a valuable tool to investigate the dynamics of polariton build-up, along with the possibility to generate non-classical states of light via the mechanism of dynamic Casimir radiation at room temperature ${ }^{41,42}$. The devices presented in this work also holds good promise as ultrafast $\mathrm{THz}$ amplitude modulators with picosecond modulation speed. 


\section{Acknowledgments:}

We warmly thank I. Carusotto for useful discussions. We acknowledge financial support from the European Union FET-Open Grant MIRBOSE (737017) and from the French National Research Agency (project ANR-18-CE24-0013 "TERASEL" and ANR-17-CE24-0016 "IRENA"). This work was partially supported by the French RENATECH network.

\section{Corresponding author:}

*email: jean-michel.manceau@c2n.upsaclay.fr

\section{Presently at:}

\# ICFO-Institut de Ciencies Fotoniques, The Barcelona Institute of Science and Technology, 08860 Castelldefels, Spain

+ Institut d’Electronique et des Systèmes, CNRS (UMR 5214), Université de Montpellier, 34095 Montpellier Cedex 5, France

\section{References:}

(1) Smith, D. R.; Padilla, W. J.; Vier, D. C.; Nemat-Nasser, S. C.; Schultz, S. Composite Medium with Simultaneously Negative Permeability and Permittivity. Phys. Rev. Lett. 2000, 84 (18), 41844187. https://doi.org/10.1103/PhysRevLett.84.4184.

(2) Shelby, R. A.; Smith, D. R.; Schultz, S. Experimental Verification of a Negative Index of Refraction. Science 2001, 292 (5514), 77. https://doi.org/10.1126/science.1058847.

(3) Schurig, D.; Mock, J. J.; Justice, B. J.; Cummer, S. A.; Pendry, J. B.; Starr, A. F.; Smith, D. R. Metamaterial Electromagnetic Cloak at Microwave Frequencies. Science 2006, 314 (5801), 977. https://doi.org/10.1126/science.1133628.

(4) Zheludev, N. I.; Kivshar, Y. S. From Metamaterials to Metadevices. Nature Mater 2012, 11 (11), 917-924. https://doi.org/10.1038/nmat3431.

(5) Padilla, W. J.; Taylor, A. J.; Highstrete, C.; Lee, M.; Averitt, R. D. Dynamical Electric and Magnetic Metamaterial Response at Terahertz Frequencies. Phys. Rev. Lett. 2006, 96 (10), 107401. https://doi.org/10.1103/PhysRevLett.96.107401.

(6) Chen, H.-T.; O'Hara, J. F.; Azad, A. K.; Taylor, A. J.; Averitt, R. D.; Shrekenhamer, D. B.; Padilla, W. J. Experimental Demonstration of Frequency-Agile Terahertz Metamaterials. Nature Photon 2008, 2 (5), 295-298. https://doi.org/10.1038/nphoton.2008.52.

(7) Manceau, J.-M.; Shen, N.-H.; Kafesaki, M.; Soukoulis, C. M.; Tzortzakis, S. Dynamic Response of Metamaterials in the Terahertz Regime: Blueshift Tunability and Broadband Phase Modulation. Appl. Phys. Lett. 2010, 96 (2), 021111. https://doi.org/10.1063/1.3292208.

(8) Shen, N.-H.; Massaouti, M.; Gokkavas, M.; Manceau, J.-M.; Ozbay, E.; Kafesaki, M.; Koschny, T.; Tzortzakis, S.; Soukoulis, C. M. Optically Implemented Broadband Blueshift Switch in the Terahertz Regime. Phys. Rev. Lett. 2011, 106 (3), 037403. https://doi.org/10.1103/PhysRevLett.106.037403.

(9) Wang, L.; Zhang, Y.; Guo, X.; Chen, T.; Liang, H.; Hao, X.; Hou, X.; Kou, W.; Zhao, Y.; Zhou, T.; Liang, S.; Yang, Z. A Review of THz Modulators with Dynamic Tunable Metasurfaces. Nanomaterials 2019, 9 (7), 965. https://doi.org/10.3390/nano9070965.

(10) Geiser, M.; Scalari, G.; Castellano, F.; Beck, M.; Faist, J. Room Temperature Terahertz Polariton Emitter. Appl. Phys. Lett. 2012, 101 (14), 141118. https://doi.org/10.1063/1.4757611.

(11) Lee, J.; Jung, S.; Chen, P.-Y.; Lu, F.; Demmerle, F.; Boehm, G.; Amann, M.-C.; Alù, A.; Belkin, M. A. Ultrafast Electrically Tunable Polaritonic Metasurfaces. Advanced Optical Materials 2014, 2 (11), 1057-1063. https://doi.org/10.1002/adom.201400185. 
(12) Lee, J.; Tymchenko, M.; Argyropoulos, C.; Chen, P.-Y.; Lu, F.; Demmerle, F.; Boehm, G.; Amann, M.-C.; Alù, A.; Belkin, M. A. Giant Nonlinear Response from Plasmonic Metasurfaces Coupled to Intersubband Transitions. Nature 2014, 511 (7507), 65-69.

https://doi.org/10.1038/nature13455.

(13) Benz, A.; Krall, M.; Schwarz, S.; Dietze, D.; Detz, H.; Andrews, A. M.; Schrenk, W.; Strasser, G.; Unterrainer, K. Resonant Metamaterial Detectors Based on THz Quantum-Cascade Structures. Sci Rep 2015, 4 (1), 4269. https://doi.org/10.1038/srep04269.

(14) Paulillo, B.; Pirotta, S.; Nong, H.; Crozat, P.; Guilet, S.; Xu, G.; Dhillon, S.; Li, L. H.; Davies, A. G.; Linfield, E. H.; Colombelli, R. Ultrafast Terahertz Detectors Based on Three-Dimensional MetaAtoms. Optica 2017, 4 (12), 1451. https://doi.org/10.1364/OPTICA.4.001451.

(15) Palaferri, D.; Todorov, Y.; Bigioli, A.; Mottaghizadeh, A.; Gacemi, D.; Calabrese, A.; Vasanelli, A.; Li, L.; Davies, A. G.; Linfield, E. H.; Kapsalidis, F.; Beck, M.; Faist, J.; Sirtori, C. RoomTemperature Nine-Mm-Wavelength Photodetectors and GHz-Frequency Heterodyne Receivers. Nature 2018, 556 (7699), 85-88. https://doi.org/10.1038/nature25790.

(16) Todorov, Y.; Sirtori, C. Few-Electron Ultrastrong Light-Matter Coupling in a Quantum LC Circuit. Phys. Rev. X 2014, 4 (4), 041031. https://doi.org/10.1103/PhysRevX.4.041031.

(17) Ciuti, C.; Bastard, G.; Carusotto, I. Quantum Vacuum Properties of the Intersubband Cavity Polariton Field. Phys. Rev. B 2005, 72 (11), 115303.

https://doi.org/10.1103/PhysRevB.72.115303.

(18) Liberato, S. D.; Ciuti, C.; Carusotto, I. Quantum Vacuum Radiation Spectra from a Semiconductor Microcavity with a Time-Modulated Vacuum Rabi Frequency. Phys. Rev. Lett. 2007, 98 (10), 103602. https://doi.org/10.1103/PhysRevLett.98.103602.

(19) Frisk Kockum, A.; Miranowicz, A.; De Liberato, S.; Savasta, S.; Nori, F. Ultrastrong Coupling between Light and Matter. Nat Rev Phys 2019, 1 (1), 19-40. https://doi.org/10.1038/s42254018-0006-2.

(20) Deimert, C.; Goulain, P.; Manceau, J.-M.; Pasek, W.; Yoon, T.; Bousseksou, A.; Kim, N. Y.; Colombelli, R.; Wasilewski, Z. R. Realization of Harmonic Oscillator Arrays with Graded Semiconductor Quantum Wells. Phys. Rev. Lett. 2020, 125 (9), 097403. https://doi.org/10.1103/PhysRevLett.125.097403.

(21) Scalari, G.; Maissen, C.; Turcinkova, D.; Hagenmuller, D.; De Liberato, S.; Ciuti, C.; Reichl, C.; Schuh, D.; Wegscheider, W.; Beck, M.; Faist, J. Ultrastrong Coupling of the Cyclotron Transition of a 2D Electron Gas to a THz Metamaterial. Science 2012, 335 (6074), 1323-1326. https://doi.org/10.1126/science.1216022.

(22) Halbhuber, M.; Mornhinweg, J.; Zeller, V.; Ciuti, C.; Bougeard, D.; Huber, R.; Lange, C. NonAdiabatic Stripping of a Cavity Field from Electrons in the Deep-Strong Coupling Regime. Nature Photonics 2020, 14 (11), 675-679. https://doi.org/10.1038/s41566-020-0673-2.

(23) Fan, K.; Strikwerda, A. C.; Zhang, X.; Averitt, R. D. Three-Dimensional Broadband Tunable Terahertz Metamaterials. Phys. Rev. B 2013, 87 (16), 161104. https://doi.org/10.1103/PhysRevB.87.161104.

(24) Paulillo, B.; Manceau, J. M.; Degiron, A.; Zerounian, N.; Beaudoin, G.; Sagnes, I.; Colombelli, R. Circuit-Tunable Sub-Wavelength THz Resonators: Hybridizing Optical Cavities and Loop Antennas. Opt. Express 2014, 22 (18), 21302. https://doi.org/10.1364/OE.22.021302.

(25) Malerba, M.; Alabastri, A.; Miele, E.; Zilio, P.; Patrini, M.; Bajoni, D.; Messina, G. C.; Dipalo, M.; Toma, A.; Proietti Zaccaria, R.; De Angelis, F. 3D Vertical Nanostructures for Enhanced Infrared Plasmonics. Scientific Reports 2015, 5 (1), 16436. https://doi.org/10.1038/srep16436.

(26) Todorov, Y.; Desfonds, P.; Belacel, C.; Becerra, L.; Sirtori, C. Three-Dimensional THz LumpedCircuit Resonators. Opt. Express 2015, 23 (13), 16838. https://doi.org/10.1364/OE.23.016838.

(27) Paulillo, B.; Manceau, J.-M.; Li, L. H.; Davies, A. G.; Linfield, E. H.; Colombelli, R. Room Temperature Strong Light-Matter Coupling in Three Dimensional Terahertz Meta-Atoms. Appl. Phys. Lett. 2016, 108 (10), 101101. https://doi.org/10.1063/1.4943167.

(28) Malerba, M.; Ongarello, T.; Paulillo, B.; Manceau, J.-M.; Beaudoin, G.; Sagnes, I.; De Angelis, F.; Colombelli, R. Towards Strong Light-Matter Coupling at the Single-Resonator Level with Sub- 
Wavelength Mid-Infrared Nano-Antennas. Appl. Phys. Lett. 2016, 109 (2), 021111.

https://doi.org/10.1063/1.4958330.

(29) Jeannin, M.; Mariotti Nesurini, G.; Suffit, S.; Gacemi, D.; Vasanelli, A.; Li, L.; Davies, A. G.; Linfield, E.; Sirtori, C.; Todorov, Y. Ultrastrong Light-Matter Coupling in Deeply Subwavelength THz LC Resonators. ACS Photonics 2019, 6 (5), 1207-1215.

https://doi.org/10.1021/acsphotonics.8b01778.

(30) Chen, H.-T.; Padilla, W. J.; Zide, J. M. O.; Bank, S. R.; Gossard, A. C.; Taylor, A. J.; Averitt, R. D. Ultrafast Optical Switching of Terahertz Metamaterials Fabricated on ErAs/GaAs Nanoisland Superlattices. Opt. Lett. 2007, 32 (12), 1620. https://doi.org/10.1364/OL.32.001620.

(31) Liu, P. Q.; Luxmoore, I. J.; Mikhailov, S. A.; Savostianova, N. A.; Valmorra, F.; Faist, J.; Nash, G. R. Highly Tunable Hybrid Metamaterials Employing Split-Ring Resonators Strongly Coupled to Graphene Surface Plasmons. Nat Commun 2015, 6 (1), 8969. https://doi.org/10.1038/ncomms9969.

(32) Hu, Y.; Jiang, T.; Zhou, J.; Hao, H.; Sun, H.; Ouyang, H.; Tong, M.; Tang, Y.; Li, H.; You, J.; Zheng, X.; Xu, Z.; Cheng, X. Ultrafast Terahertz Frequency and Phase Tuning by All-Optical Molecularization of Metasurfaces. Adv. Optical Mater. 2019, 7 (22), 1901050. https://doi.org/10.1002/adom.201901050.

(33) Hu, Y.; Tong, M.; Cheng, X.; Zhang, J.; Hao, H.; You, J.; Zheng, X.; Jiang, T. Bi2Se3Functionalized Metasurfaces for Ultrafast All-Optical Switching and Efficient Modulation of Terahertz Waves. ACS Photonics 2021. https://doi.org/10.1021/acsphotonics.0c01194.

(34) Beard, M. C.; Turner, G. M.; Schmuttenmaer, C. A. Subpicosecond Carrier Dynamics in LowTemperature Grown GaAs as Measured by Time-Resolved Terahertz Spectroscopy. Journal of Applied Physics 2001, 90 (12), 5915-5923. https://doi.org/10.1063/1.1416140.

(35) Gregory, I. S.; Baker, C.; Tribe, W. R.; Evans, M. J.; Beere, H. E.; Linfield, E. H.; Davies, A. G.; Missous, M. High Resistivity Annealed Low-Temperature GaAs with 100 Fs Lifetimes. Appl. Phys. Lett. 2003, 83 (20), 4199-4201. https://doi.org/10.1063/1.1628389.

(36) Bacon, D. R.; Burnett, A. D.; Swithenbank, M.; Russell, C.; Li, L.; Wood, C. D.; Cunningham, J.; Linfield, E. H.; Davies, A. G.; Dean, P.; Freeman, J. R. Free-Space Terahertz Radiation from a LTGaAs-on-Quartz Large-Area Photoconductive Emitter. Opt. Express 2016, 24 (23), 26986. https://doi.org/10.1364/OE.24.026986.

(37) Maussang, K.; Palomo, J.; Manceau, J.-M.; Colombelli, R.; Sagnes, I.; Li, L. H.; Linfield, E. H.; Davies, A. G.; Mangeney, J.; Tignon, J.; Dhillon, S. S. Monolithic Echo-Less Photoconductive Switches as a High-Resolution Detector for Terahertz Time-Domain Spectroscopy. Appl. Phys. Lett. 2017, 110 (14), 141102. https://doi.org/10.1063/1.4979536.

(38) Koulouklidis, A. D.; Fedorov, V. Yu.; Tzortzakis, S. Spectral Bandwidth Scaling Laws and Reconstruction of THz Wave Packets Generated from Two-Color Laser Plasma Filaments. Phys. Rev. A 2016, 93 (3), 033844. https://doi.org/10.1103/PhysRevA.93.033844.

(39) Karpowicz, N.; Dai, J.; Lu, X.; Chen, Y.; Yamaguchi, M.; Zhao, H.; Zhang, X.-C.; Zhang, L.; Zhang, C.; Price-Gallagher, M.; Fletcher, C.; Mamer, O.; Lesimple, A.; Johnson, K. Coherent Heterodyne Time-Domain Spectrometry Covering the Entire "Terahertz Gap." Appl. Phys. Lett. 2008, 92 (1), 011131. https://doi.org/10.1063/1.2828709.

(40) Bacon, D. R.; Bacon, D. R.; Bacon, D. R.; Gill, T. B.; Rosamond, M.; Burnett, A. D.; Dunn, A.; Li, L.; Linfield, E. H.; Davies, A. G.; Dean, P.; Freeman, J. R.; Freeman, J. R. Photoconductive Arrays on Insulating Substrates for High-Field Terahertz Generation. Opt. Express, OE 2020, 28 (12), 17219-17231. https://doi.org/10.1364/OE.391656.

(41) Liberato, S. D.; Ciuti, C.; Carusotto, I. Quantum Vacuum Radiation Spectra from a Semiconductor Microcavity with a Time-Modulated Vacuum Rabi Frequency. Phys. Rev. Lett. 2007, 98 (10), 103602. https://doi.org/10.1103/PhysRevLett.98.103602.

(42) Wilson, C. M.; Johansson, G.; Pourkabirian, A.; Simoen, M.; Johansson, J. R.; Duty, T.; Nori, F.; Delsing, P. Observation of the Dynamical Casimir Effect in a Superconducting Circuit. Nature 2011, 479 (7373), 376-379. https://doi.org/10.1038/nature10561. 


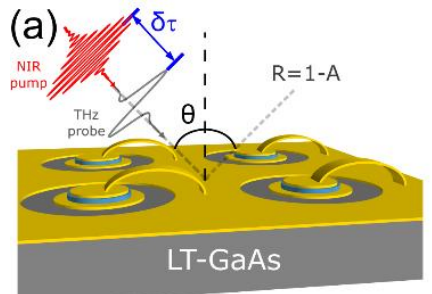

(b)

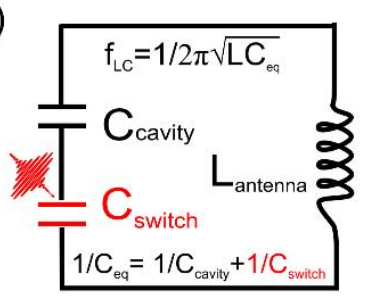

(c)

(d)
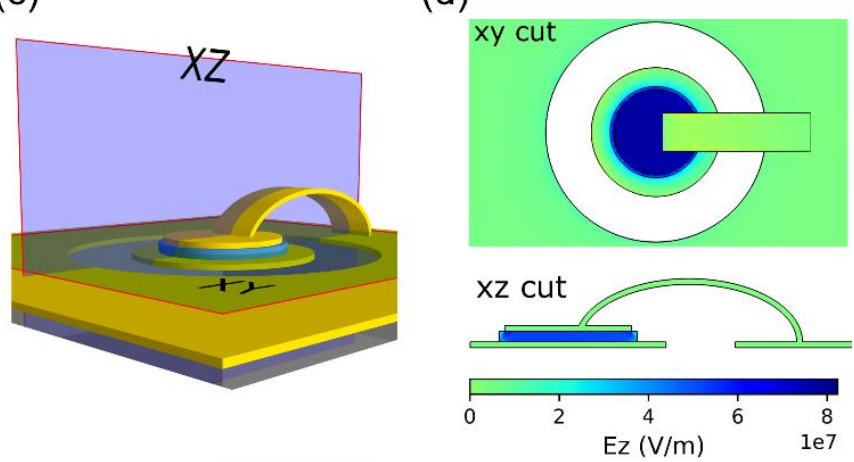

(e)

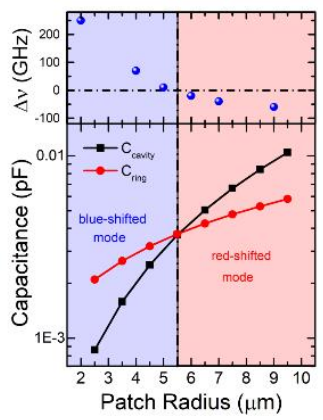

(f)

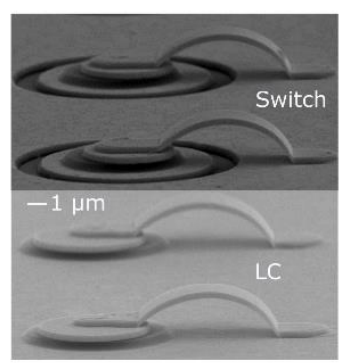

Figure 1: (a) Schematic representation of the experimental approach with the array of LC switches probed by a THz pulse and photo-excited by a synchronized NIR pulse. (b) Equivalent circuit of the device (c) Schematic representation of a single LC-switch resonator with two planes cut represented (d) Simulated confined electric field in the $z$ direction for both plane cuts. (e) Calculated capacitance value of the ring and the cavity as function of the patch radius. The ring opening size is kept constant at $1.4 \mu \mathrm{m}$. (f) Scanning Electron Microscope picture of the fabricated device. 

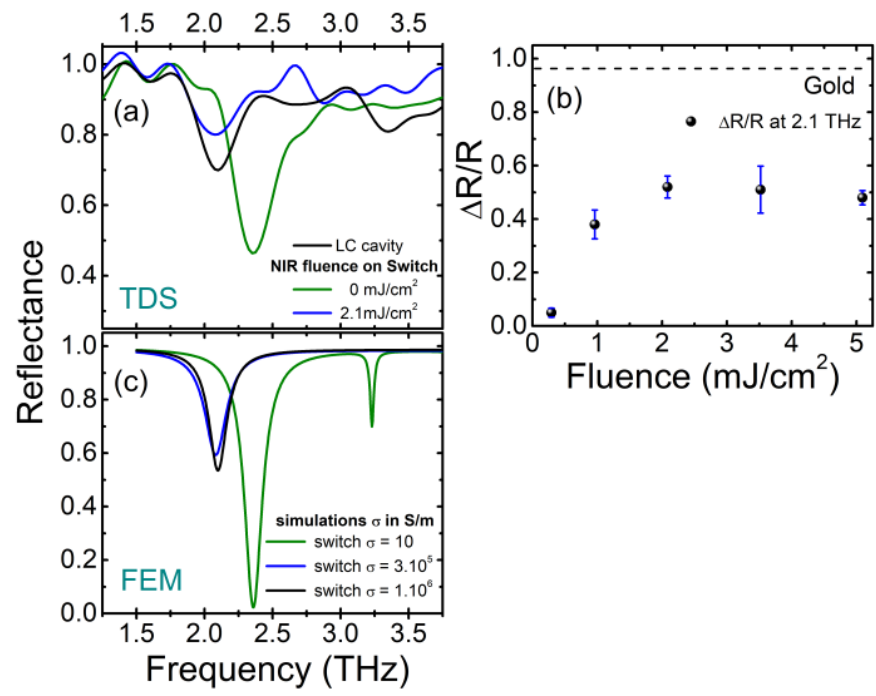

Figure 2: (a) Reflectance of the LC resonator (black line) with TDS technique at 45 degrees incidence along with the reflectance of the LC switch with and without illumination (green/ blue curves). A 280 $\mathrm{GHz}$ shift is experimentally observed between the natural eigen mode (green) and the photo-activated one under $2.1 \mathrm{~mJ} / \mathrm{cm}^{2}$ of NIR illumination (blue). (b) Relative reflectivity $\left(\Delta R / R=\left(R_{\text {illu. }}-R_{\text {dark }}\right) / R_{\text {dark }}\right)$ of a bare LT-GaAs sample at different fluence and for a frequency of $2.1 \mathrm{THz}$. The relative change of reflectivity saturates at a fluence of $2.1 \mathrm{~mJ} / \mathrm{cm}^{2}$, a value nearly twice smaller than the one of a metallic surface. (c) FEM simulations of the switch device for different value of conductivity (in $\mathrm{S} / \mathrm{m}$ ) within the substrate. Note the good agreement with the experimental value presented above. 

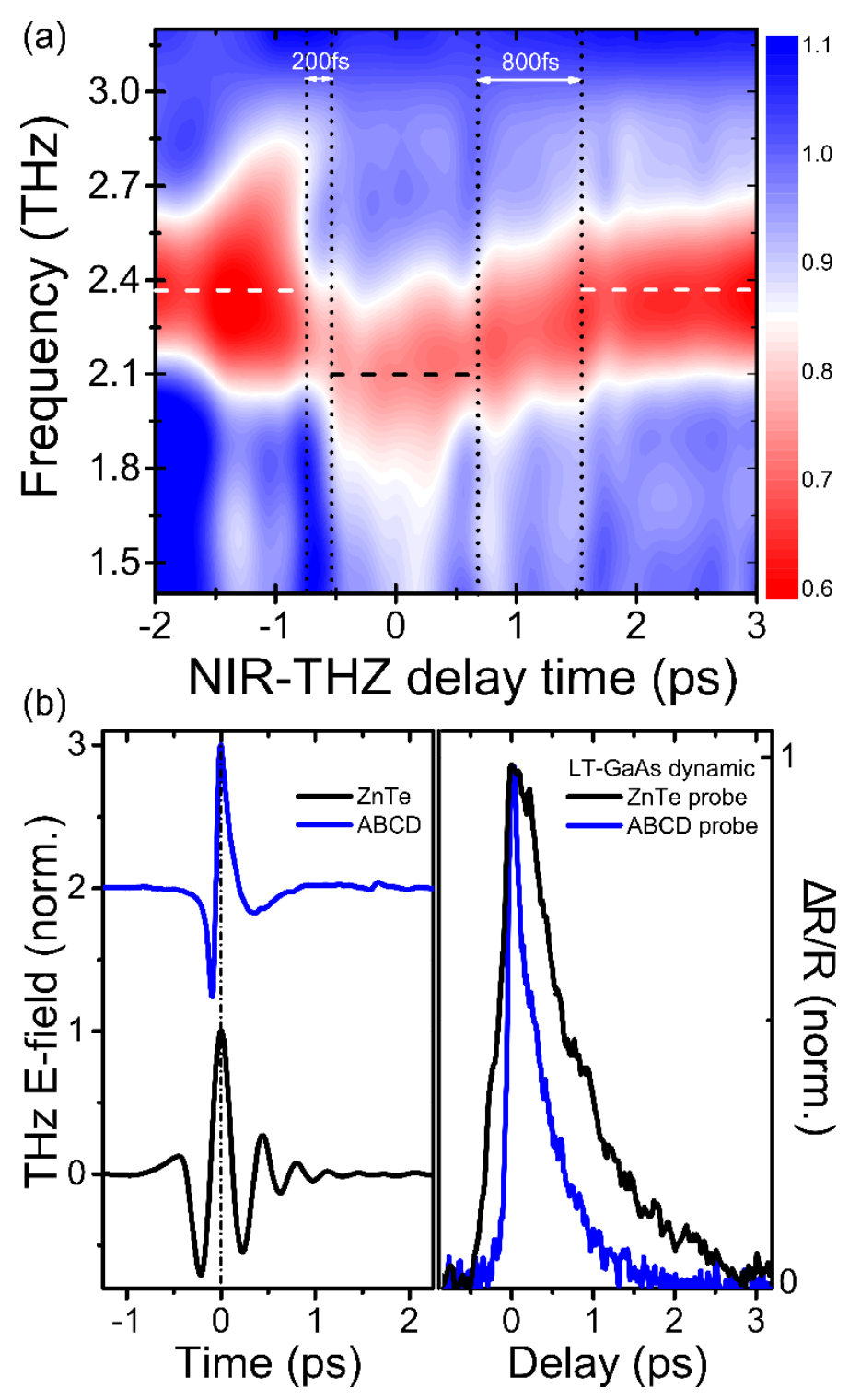

Figure 3: (a) Two-dimensional map of the spectral reflectance as a function of the time delay between the $\mathrm{THz}$ pulse and the NIR pulse. The initial frequency is depicted with white dashed lines while the switched mode with a black one. Note the sub-ps time scale of the switch ON and OFF towards the photoactivated mode. This mode remains active for a duration of $1.2 \mathrm{ps}$. (b) bare temporal traces of the $\mathrm{THz}$ electric field recorded with electro-optic (ZnTe) and air biased (ABCD) sampling techniques. Right panel. Carriers dynamic within the bare LT-GaAs wafer as a function of the time delay with the NIR pump pulse recorded with both techniques. 